\title{
Functional characterization of two defensins, HIDFS1 and HIDFS2, from the hard tick Haemaphysalis longicornis
}

Ta Sun $^{1 \dagger}$, Wen Pan ${ }^{1 \dagger}$, Yanhui Song ${ }^{2}$, Jingpin Zhang ${ }^{1}$, Jingwen Wang ${ }^{3^{*}}$ and Jianfeng Dai ${ }^{1 *}$ (D)

\begin{abstract}
Background: Ticks are second to mosquitoes as vectors of human arthropod-borne diseases. Ticks rely heavily on antimicrobial peptides (AMPs) to defend against microbes and defensins are major components of innate immunity in ticks.

Results: Two novel defensin genes, named HIDFS1 and HIDFS2, were identified from a CDNA library of the hard tick Haemaphysalis longicornis collected in southeast China. The peptides encoded by both genes shares typical features of type-2 arthropod defensin superfamily. The expressions of both genes increased in ticks during blood-feeding. The synthetic minimum functional peptides HIDFS1 and HIDFS2 showed broad spectrum antimicrobial activity against various Gram-positive and Gram-negative bacteria. Moreover, HIDFS1 and HIDFS2 exhibit bactericidal activity to some drug resistant bacteria. HIDFS1, but not HIDFS2, showed inhibitory activity against fungus Candida albicans. HIDFS1 and HIDFS2 had no significant hemolysis effect on human erythrocytes at low concentrations and did not impair mammalian cell survival. Finally, HIDFS1 and HIDFS2 significantly protected mice against lethal infection by Staphylococcus aureus and Micrococcus luteus.

Conclusions: HIDFS1 and HIDFS2 are two novel functional defensins from the hard tick Haemaphysalis longicornis. They showed bactericidal activity against various Gram-positive and Gram-negative bacteria and significantly protect mice against lethal bacterial infection. Thus, HIDFS1 and HIDFS2 can be introduced to the medical field as new drug candidates with antibacterial activity.
\end{abstract}

Keywords: Defensin, Antimicrobial peptide (AMP), Antibiotic resistant bacteria, Tick, Haemaphysalis longicornis

\section{Introduction}

Ticks are obligate hematophagous ectoparasites, living by feeding on the blood of mammals, birds, and sometimes reptiles and amphibians [1]. Ticks harm people indirectly by transmitting pathogenic organisms, such as protozoa, viruses and bacteria. As they are widely distributed around the world, especially in warm, humid climates, ticks occupy the second place, after mosquitoes, in the abundance of transmission of arthropodborne diseases. The typical tick-borne diseases include

\footnotetext{
* Correspondence: jingwenwang@fudan.edu.cn; daijianfeng@suda.edu.cn

${ }^{\dagger}$ Equal contributors

${ }^{3}$ School of Life Science, Fudan University, Shanghai, People's Republic of China

${ }^{1}$ Institutes of Biology and Medical Sciences, Jiangsu Key Laboratory of Infection and Immunity, Soochow University, Suzhou, People's Republic of China

Full list of author information is available at the end of the article
}

tick-borne encephalitis, Crimean-Congo hemorrhagic fever, Lyme disease, Q fever and Rocky Mountain spotted fever, among others $[1,2]$.

Lacking lymphocytes, thymuses, or antibodies, ticks rely heavily on antimicrobial peptides (AMPs) to defend against microbes, so that they can live harmoniously with microbes [3-5]. Among the identified and widely characterized tick AMPs, defensins and their isoforms have been identified in many tick species including Amblyomma americanum, Dermacentor variabilis, Haemaphysalis longicornis, Ixodes scapularis, Ixodes ricinus, Ornithodoros moubata and Rhipicephalus microplus [6-9]. Defensins are small cysteinerich cationic proteins (usually contain six cysteine residues) forming disulfide bridges with the conserved pairing Cys1Cys4, Cys2-Cys5, and Cys3-Cys6 [3, 6, 10]. They are usually expressed in the midgut (MG) after blood-feeding and pathogen invasion [11]. For examples, hemocoelic 
inoculations with Borrelia burgdorferi of D. variabilis induced upregulation of a lysozyme-like peptide and the secretion of defensin $[12,13]$. The expressions of a 5.3-kd defensin family were upregulated upon tick acquisition of Anaplasma phagocytophilum [14]. Tick defensins have a broad spectrum of antimicrobial profiles, which are primarily directed against Gram-positive bacteria, but some isoforms are also effective against Gram-negative bacteria, viruses, fungi and protozoa $[6,15,16]$.

In this study, we characterized two defensin genes from a cDNA library of $H$. longicornis. This hard tick species was collected in the field of Zhejiang Province, located in southeast China. Lyme spirochetes, spotted fever group rickettsiae, as well as Ehrlichia chaffeensis and Anaplasma bovis, have been detected in $H$. longicornis [17, 18]. A newly identified tick-borne virus, SFTS (Severe fever with thrombocytopenia syndrome virus, fatality rates ranging between 12 and $30 \%$ in some areas), has also been shown to be transmitted via $H$. longicornis $[19,20]$.

\section{Methods}

\section{RNA sequencing and gene identification}

Total RNA from whole adult ticks $H$. longicornis were prepared and subjected to high throughput RNA sequencing. A total of 43,419 pieces of Unigene sequences were obtained and screened for novel defensing-like genes by BLAST analyses against the NCBI NR database (https://blast.ncbi.nlm.nih.gov/Blast.cgi).

\section{Gene expression}

RNA was extracted from whole adult ticks before and after tick feeding for $72 \mathrm{~h}$ on a Specific Pathogen Free (SPF) mouse. Transcript expression analysis of HIDFS1 and HIDFS2 genes was conducted by reverse transcription polymerase chain reaction (RT-PCR) using HIDFS1 and HIDFS2 gene specific primers (see primer sequences in Additional file 1: Table S1).

\section{Antimicrobial assay}

To test the antimicrobial activity of HIDFS1 and HIDFS2, the minimum functional segments of HIDFS1 (a 37 amino acid peptide) and HIDFS2 (a 36 amino acid peptide) were synthesized and purified by high-performance liquid chromatography (the final purity of peptides are > 90\%) (GL Biochem, Shanghai, China). The peptides were dissolved in PBS buffer containing $0.05 \%$ Tween 20 and $1 \mu \mathrm{M} \beta$ merkaptoethanol (at a stock concentration of $1000 \mu \mathrm{M}$ ), and diluted properly in PBS when used in the antimicrobial assay. The target bacteria used in the bactericidal assay were obtained from China Veterinary Culture Collection Center (CVCC) and operated with standard protocols. The antimicrobial assays were performed as described previously [7]. Briefly, microbial strains were grown to an OD600 nm of $0.4-0.6$ at $37^{\circ} \mathrm{C}$ in Poor Broth media $(1 \% w / v$ tryptone and $0.5 \% w / v \mathrm{NaCl}$ ) (except for Mycobacterium bovis, which was grown in Middlebrook 7H9 Broth (BD-Difco, Franklin Lakes, USA) with carbenicillin, Candida albicans which was grown in Sabouraud's Dextrose Broth (1\% w/v tryptone, $4 \% \mathrm{w} / \mathrm{v}$ glucose) and $B$. burgdorferi which was grown in complete BSK media (Sigma, St. Louis, USA) [21]. Approximately $90 \mu \mathrm{l}$ of inocula of microbial strains (diluted with $\mathrm{PB}$ media to an OD600 nm of 0.001 ) were incubated with $10 \mu \mathrm{l}$ of various concentrations of HIDFS1 and HIDFS2 $(0.1-50 \mu \mathrm{M})$ in wells of a 96-well plate. The mixture was grown for $20 \mathrm{~h}$ (for M. bovis, incubated for $48 \mathrm{~h}$ ) at $37{ }^{\circ} \mathrm{C}$ and $250 \mathrm{rpm}$. Antimicrobial activity was evaluated by measuring the absorbance of the bacterial culture at $595 \mathrm{~nm}$. Borrelia burgdorferi 297-GFP strain is an engineered spirochetes that steadily expressing GFP [22]. The anti-borrelia activities of HIDFS1 and HIDFS2 were analyzed by amplifying the flaB DNA copies in the culture media and visualizing GFP signals under a fluorescence microscope.

Eleven antibiotic resistant bacteria strains were obtained from the First Affiliated Hospital of Soochow University (Suzhou, China) under institutional guidelines. The antibiotic resistant information of some of the strains is listed in Additional file 2: Table S2. The antimicrobial activity of HIDFS1 and HIDFS2 on these antibiotic resistant bacteria were performed according to the protocols above.

\section{Hemolysis and cytotoxic assay}

Human erythrocytes hemolysis assay was performed as previously described. $0-50 \mu \mathrm{M}$ of HIDFS1 and HIDFS2 were incubated with human erythrocytes for $30 \mathrm{~min}$ at $37^{\circ} \mathrm{C}$. Red blood cells were lysised by $0.4 \%$ TritonX 100 (Sigma, St. Louis, USA) and loaded as positive controls. To test whether HIDFS1 and HIDFS2 inhibit mammalian cell proliferation, $20 \mu \mathrm{M}$ of HIDFS1 and HIDFS2 were added into the cell media of mammalian cell line A549, 293 T, K562 and THP1 and incubated for $24 \mathrm{~h}$. The cell viability assay was determined by Cell Viability Assay (Promega, Fitchburg, USA).

\section{Mice infection and protection assay}

Mice were infected with lethal doses of Micrococcus luteus $\left(1 \times 10^{6}\right.$ cfu per $\mathrm{C} 3 \mathrm{H} / \mathrm{HeJ}$ mouse $)$ and Staphylococcus aureus $\left(1 \times 10^{8} c f u\right.$ per C57/BL6 mouse) by intraperitoneal (i.p.) injection according to protocols described previously $[23,24]$. Six h after infection, mice were treated by i.p. injection of HIDFS1 (100 $\mu \mathrm{g} /$ mouse), HIDFS2 (100 $\mu \mathrm{g} /$ mouse) or saline as controls. Mice were monitored daily for survival and symptoms of disease.

\section{Results and discussion}

Isolation of defensin genes HIDFS1 and HIDFS2 from $H$. longicornis

By sequencing a cDNA library of $H$. longicornis, two cDNA clones encoding the precursor of putative defensins were 
obtained and named as HIDFS1 and HIDFS2, respectively. The cDNA and deduced amino acid sequences of HIDFS1 and HIDFS2 are shown in Fig. 1. Sequence analysis indicated that both defensin ORFs were 225 bp long, encoding 74 amino acid peptides. The predicted HIDFS1 and HIDFS2 proteins contained a putative signal peptide cleavage site at amino acid positions 18 and 23, respectively, as analyzed by SignalP4.1 software (http://www.cbs.dtu.dk/services/SignalP/). By BlastP analysis against the protein database of NCBI (https://blast.ncbi.nlm.nih.gov/Blast.cgi), the minimal critical domains of type-2 arthropod defensins, lack of the signal peptide and leading peptides, were also predicted in both HIDFSs (Fig. 1). The sequences of HIDFS1 and HIDFS2 cDNA have been deposited in the GenBank database under the accession numbers KY113087 and KY113088.

\section{Homology analysis and mRNA expression of HIDFS1 and HIDFS2}

Sequence alignment and phylogenetic analysis were conducted as described previously [7] to explore the evolutionary relationships between HIDFS1, HIDFS2 and other defensin genes in invertebrate species (Fig. 2a, b). The analysis was performed using multiple sequence alignment software ClustalW (http://www.genome.jp/tools-bin/clustalw). The results suggest that HIDFS1 and HIDFS2 shared high similarity to defensins from hard tick species, such as H. longicornis (vs BAD93183.1, 52.7\% and 67.6\%, respectively) $R$. microplus (65\% and 73\%, respectively), D. silvarum (54\% and $77 \%$, respectively), I. persulcatus (64\% and $67 \%$, respectively), $I$. ovatus (59\% and $64 \%$, respectively) and $A$. americanum (53\% and 55\%, respectively), and lower similarity to defensins from soft ticks, such as O. papillipes ( $45 \%$ and $47 \%$, respectively), O. rostratus ( $45 \%$ and $46 \%$, respectively) and C. puertoricensis (48\% and $46 \%$, respectively). All these defensins contained six conserved cysteine residues, including defensins from fruit fly (Drosophila melanogaster) or marine mollusk species (Crassostrea gigas and Ruditapes philippinarum) (Fig. 2a), suggesting these amino acids were critical for their function [9].

To investigate the mRNA expression of HIDFS1 and HIDFS2 during tick feeding, RNA was extracted from whole adult ticks before and after tick feeding on a clean mouse. Transcript expression analysis of HIDFS1 and

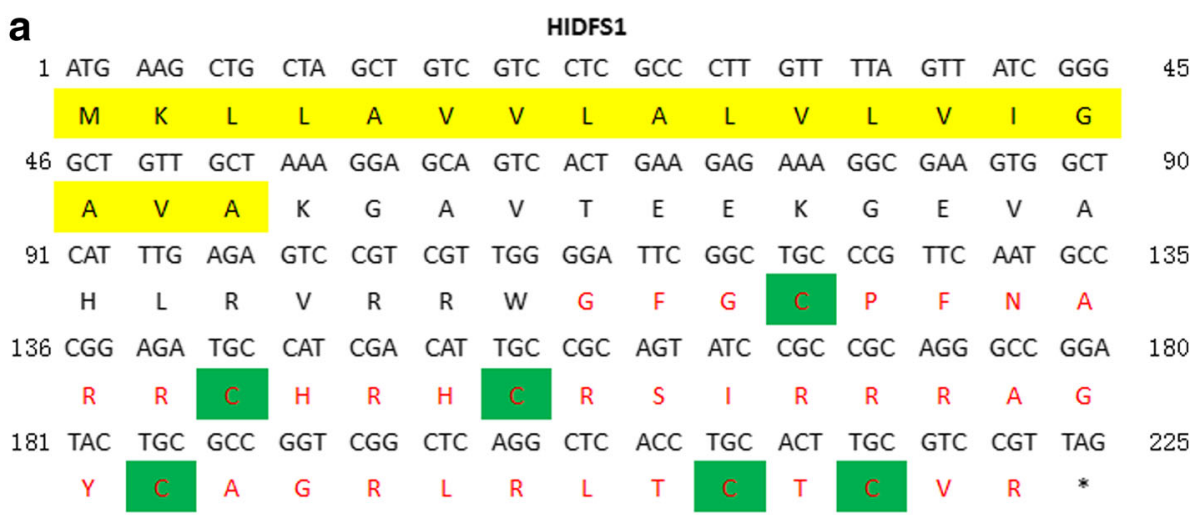

Fig. 1 Nucleotide and deduced amino acid sequences of HIDFS1 (a) and HIDFS2 (b). The signal peptide sequence is shaded in yellow. The red lettering indicates the minimum functional segment, and the green shadow indicates the location of the conserved cysteine residues found in tick defensins. The stop codons were indicated by an asterisk 


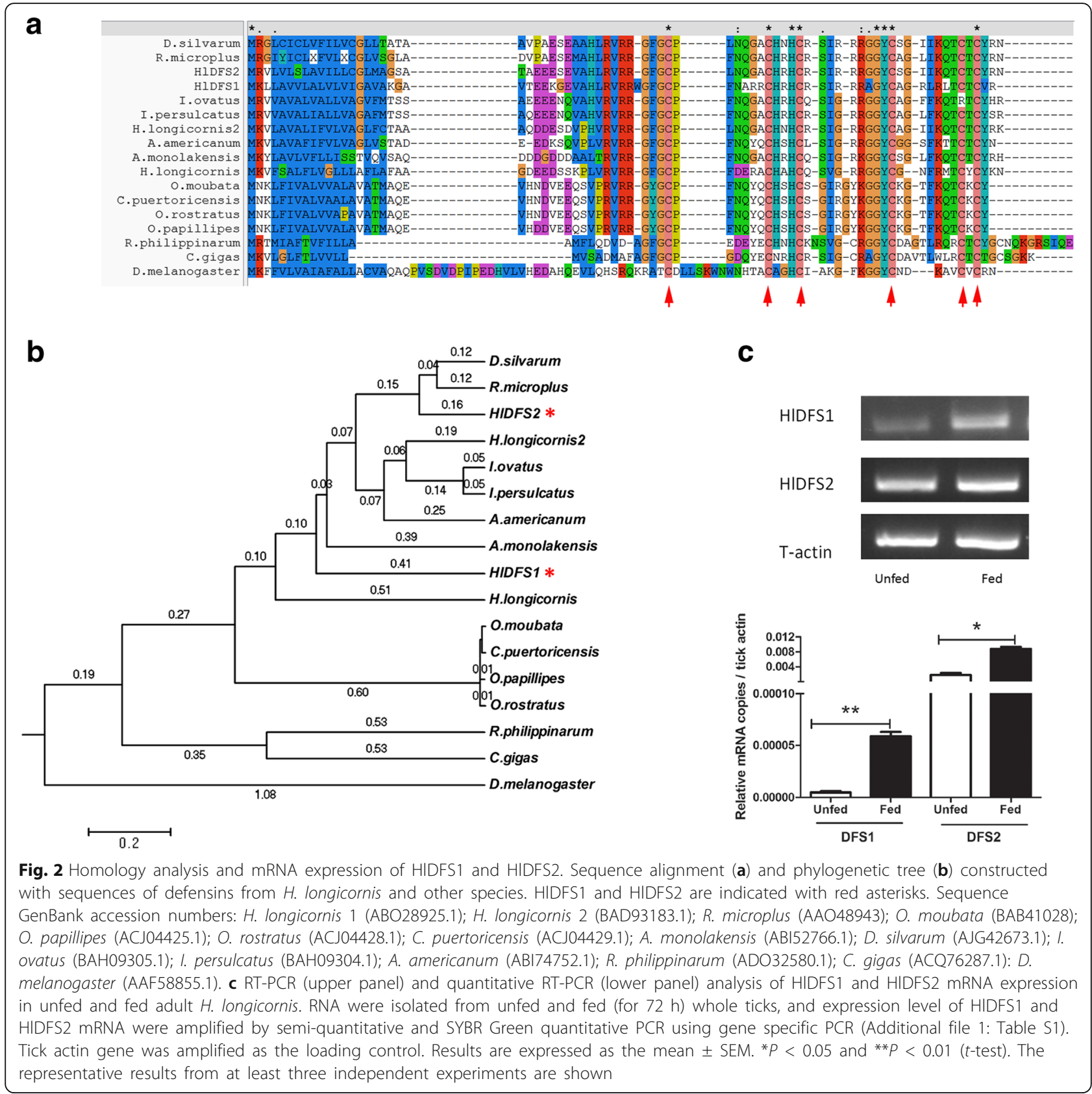

HIDFS2 genes was conducted by quantitative RT-PCR using HIDFS1 and HIDFS2 gene specific primers (see Additional file 1: Table S1). As shown in Fig. 2c, the expression levels of HIDFS1 and HIDFS2 were significantly higher in fed ticks than in unfed ticks, suggesting they are induced during tick feeding.

\section{Antimicrobial profiles of HIDFS1 and HIDFS2}

The target bacteria used in the bactericidal assay were obtained from China Veterinary Culture Collection Center (CVCC) and operated with standard protocols. Gram-positive bacteria, Bacillus pumilus (CMCC63202),
S. aureus (CMCC26003), M. luteus (CMCC28001), and M. bovis; Gram-negative bacteria, Salmonella typhimurium (CVCC542), Pseudomonas aeruginosa (CVCC2000), Escherichia coli (CMCC44103), and B. burgdorferi (297GFP strain); and fungus C. albicans (CAU0037), were used in this study. The antimicrobial assays were performed as described previously [7]. As shown in Table 1, synthetic HIDFS1 and HIDFS2 showed bactericidal activities against three Gram-positive bacteria (M. bovis, $M$. luteus and $S$. aureus). The minimal inhibitory concertation of HIDFS1 and HIDFS2 against $M$. bovis $\left(\mathrm{MIC}_{90}\right.$ $0.5 \mu \mathrm{M}$ and $0.5 \mu \mathrm{M}$, respectively) and M. luteus ( $\mathrm{MIC}_{90}$ 
Table 1 Antimicrobial profile of HIDFS1 and HIDFS2

\begin{tabular}{|c|c|c|c|c|}
\hline \multirow[t]{2}{*}{ Strain } & \multicolumn{2}{|l|}{ HIDFS1 } & \multicolumn{2}{|l|}{ HIDFS2 } \\
\hline & $\overline{M I C_{50}(\mu M)}$ & $\mathrm{MIC}_{90}(\mu \mathrm{M})$ & $\mathrm{MIC}_{50}(\mu \mathrm{M})$ & $\mathrm{MIC}_{90}(\mu \mathrm{M})$ \\
\hline \multicolumn{5}{|l|}{ Gram-positive bacteria } \\
\hline Bacillus pumilus (CMCC63202) & No effect & No effect & No effect & No effect \\
\hline Staphylococcus aureus (CMCC26003) & 10 & 50 & 50 & $>50$ \\
\hline Micrococcus luteus (CMCC28001) & 5 & 10 & 1 & 1 \\
\hline Mycobacterium bovis & 0.2 & 0.5 & 0.5 & 0.5 \\
\hline \multicolumn{5}{|l|}{ Gram-negative bacteria } \\
\hline Salmonella typhimurium (CVCC542) & No effect & No effect & No effect & No effect \\
\hline Pseudomonas aeruginosa (CVCC2000) & No effect & No effect & No effect & No effect \\
\hline Escherichia coli (CMCC44103) & 5 & 5 & 1 & 1 \\
\hline Borrelia burgdorferi (297-GFP) & 50 & 50 & 5 & 20 \\
\hline \multicolumn{5}{|l|}{ Fungi } \\
\hline Candida albicans (CAU0037) & 50 & $>50$ & No effect & No effect \\
\hline
\end{tabular}

Abbreviations: $\mathrm{MIC}_{50}$ and $\mathrm{MIC}_{90}$ : minimum inhibitory concentration required to inhibit the growth of $50 \%\left(\mathrm{MIC}_{50}\right)$ or $90 \%$ ( $\left.\mathrm{MIC}_{90}\right)$ of organisms. Determination of MICs was performed at least three times in triplicates

$10 \mu \mathrm{M}$ and $1 \mu \mathrm{M}$, respectively) were relatively low. HIDFS1 and HIDFS2 did not affect the growth of $B$. pumilus. Unlike other defensins reported less effective to Gram-negative bacteria, HIDFS1 and HIDFS2 also had an ability to inhibit some Gram-negative bacteria, for example E. coli ( $\mathrm{MIC}_{90} 5 \mu \mathrm{M}$ and $1 \mu \mathrm{M}$, respectively) and B. burgdorferi ( $\mathrm{MIC}_{90} 50 \mu \mathrm{M}$ and $20 \mu \mathrm{M}$, respectively) (Table 1, Additional file 3: Figure S1). Since B. burgdorferi is a major human pathogen that has been reported in this tick, this data suggested the potential roles of HIDFS1 and HIDFS2 in controlling this pathogen in ticks. In addition, HIDFS1 inhibited the growth of fungus $C$. albicans with an $\mathrm{MIC}_{90}$ of $50 \mu \mathrm{M}$, while HIDFS2 did not impact the fungus growth. Both defensins showed no significant influence on vesicular stomatitis virus (VSV) replication, as determined by adding HIDFS1 and HIDFS2 into the media of $293 \mathrm{~T}$ cells infected with VSV-GFP virus (Additional file 4: Figure S2).

\section{HIDFS1 and HIDFS2 inhibit the growth of antibiotic resistant bacteria}

As drug resistance is a growing public health concern, we explored the role of HIDFS1 and HIDFS2 on eleven clinical isolated antibiotic resistant bacteria. As shown in Table 2, both HIDFS1 and HIDFS2 had significant inhibitory activities against various antibiotic resistant

Table 2 Antimicrobial activity of HIDFS1 and HIDFS2 against antibiotic resistant bacteria

\begin{tabular}{|c|c|c|c|c|}
\hline \multirow[t]{2}{*}{ Strain } & \multicolumn{2}{|l|}{ HIDFS1 } & \multicolumn{2}{|l|}{ HIDFS2 } \\
\hline & $\mathrm{MIC}_{50}(\mu \mathrm{M})$ & $\mathrm{MIC}_{90}(\mu \mathrm{M})$ & $\mathrm{MIC}_{50}(\mu \mathrm{M})$ & $\mathrm{MIC}_{90}(\mu \mathrm{M})$ \\
\hline \multicolumn{5}{|l|}{ Gram-positive bacteria } \\
\hline Staphylococcus aureus (No. 570) & 50 & $>50$ & $>50$ & $>50$ \\
\hline Staphylococcus epidermidis (No. 526) & 20 & 50 & 20 & 50 \\
\hline Staphylococcus epidermidis (No. 527) & 5 & 50 & 2 & 2 \\
\hline Staphylococcus epidermidis (No. 532) & 50 & $>50$ & No effect & No effect \\
\hline \multicolumn{5}{|l|}{ Gram-negative bacteria } \\
\hline Acinetobacter baumannii (No. 531) & 5 & 50 & 10 & 20 \\
\hline Acinetobacter baumannii (No. 546) & 50 & $>50$ & 50 & $>50$ \\
\hline Enterobacter aerogenes (No. 516) & 50 & $>50$ & $>50$ & $>50$ \\
\hline Escherichia coli (No. 572) & $>50$ & $>50$ & 50 & $>50$ \\
\hline Escherichia coli (No. 582) & 50 & $>50$ & $>50$ & $>50$ \\
\hline Klebsiella pneumoniae (No. 570) & No effect & No effect & No effect & No effect \\
\hline Klebsiella pneumoniae (No. 593) & $>50$ & $>50$ & No effect & No effect \\
\hline
\end{tabular}


Gram-positive and Gram-negative bacteria. For example, HIDSF1 and HIDFS2 inhibited antibiotic resistant Staphylococcus epidermidis strain No. 527 at an $\mathrm{MIC}_{50}$ of $2 \mu \mathrm{M}$ and $\mathrm{MIC}_{90}$ of $2 \mu \mathrm{M}$, respectively. HIDSF1 and HIDFS2 also killed drug-resistant Gram-negative Acinetobacter baumannii strain No. 531 at an $\mathrm{MIC}_{50}$ of $5 \mu \mathrm{M}$ and $10 \mu \mathrm{M}$, respectively. These data indicated that HIDFS1 and HIDFS2 have the ability to inhibit some antibiotic resistant bacteria and can be potential candidates for clinical application.

\section{HIDFS1 and HIDFS2 are not hemolytic and cytotoxic to mammalian cells}

To test whether HIDFS1 and HIDFS2 have cytotoxic effect on mammalian cells, we measured the hemolysis efficiency of both peptides in concentrations effective at killing Gram-positive bacteria. The results showed that both HIDFS1 and HIDFS2 are harmless to human erythrocytes in concentrations of up to $10 \mu \mathrm{M}$ (Fig. 3a). Furthermore, HIDFS1 and HIDFS2 showed no detectable cytotoxicity to multiple mammalian cell lines, including A549, 293 T, K562 and THP1 cells (Fig. 3b).

\section{HIDFS1 and HIDFS2 significantly protect mice against} lethal bacterial infection

Since HIDFS1 or HIDFS2 shows significant bactericidal activity against Gram-positive bacteria $M$. luteus and $S$. aureus, we tested the antimicrobial activity of HIDFS1 or HIDFS2 in vivo using mouse infection models. The results suggested that both
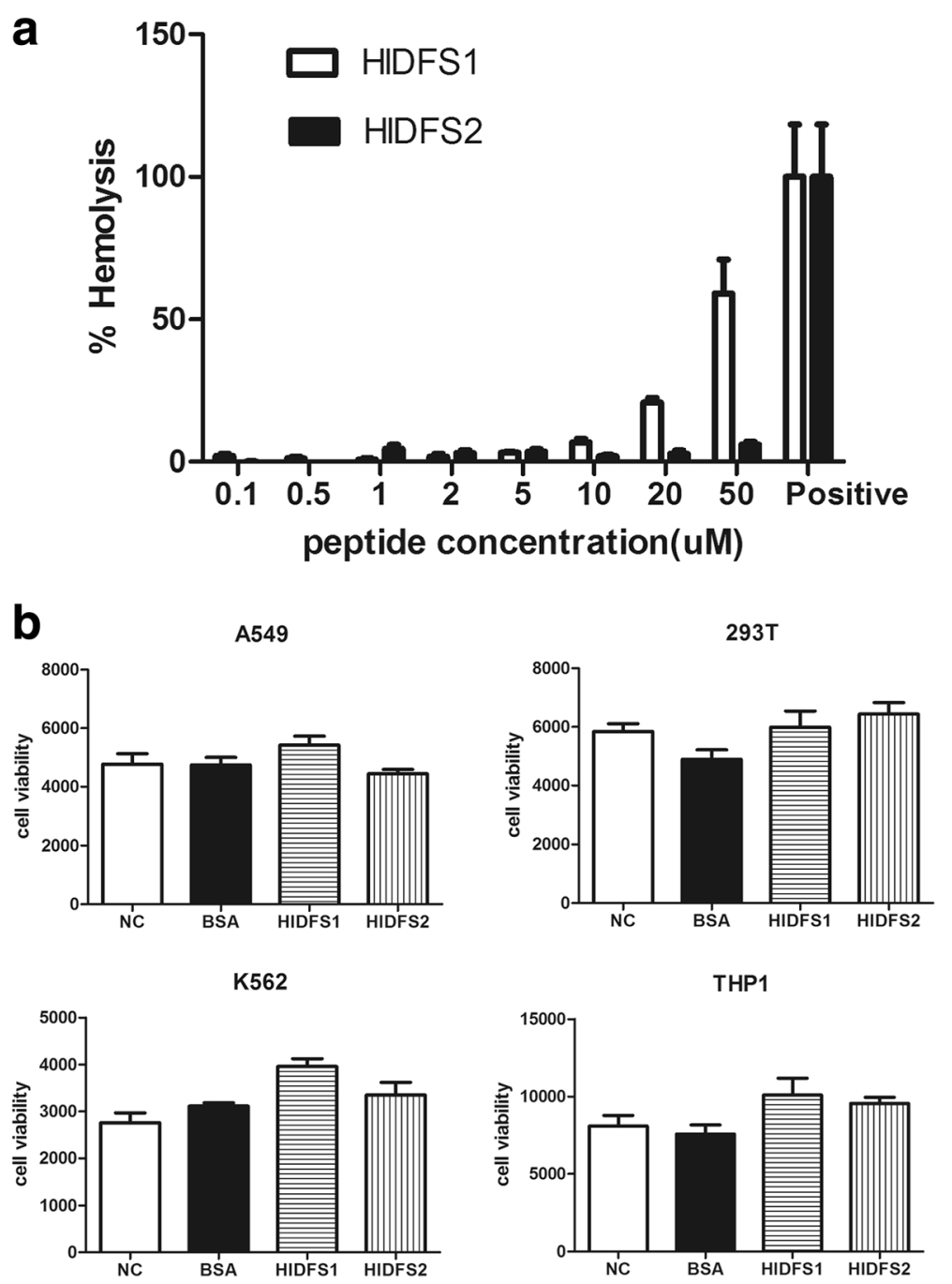

Fig. 3 Hemolytic and cytotoxic assay of HIDFS1 and HIDFS2. a HIDFSs showed no significant hemolytic effect at concentrations of $10 \mu \mathrm{M}$ (HIDFS1) and $50 \mu \mathrm{M}$ (HIDFS2). Red blood cells were lysed by $0.4 \%$ TritonX 100 and loaded as positive controls. b HIDFS1 and HIDFS2 showed no detectable cytotoxic effect on mammalian cell line A549, 293 T, K562 and THP1 at a concentration of $20 \mu \mathrm{M}$. Cell survival rates were measured by using Cell Viability Assay (Promega) according to the manufacturer's instruction. Results are expressed as the mean \pm SEM. The representative results from at least three independent experiments are shown 


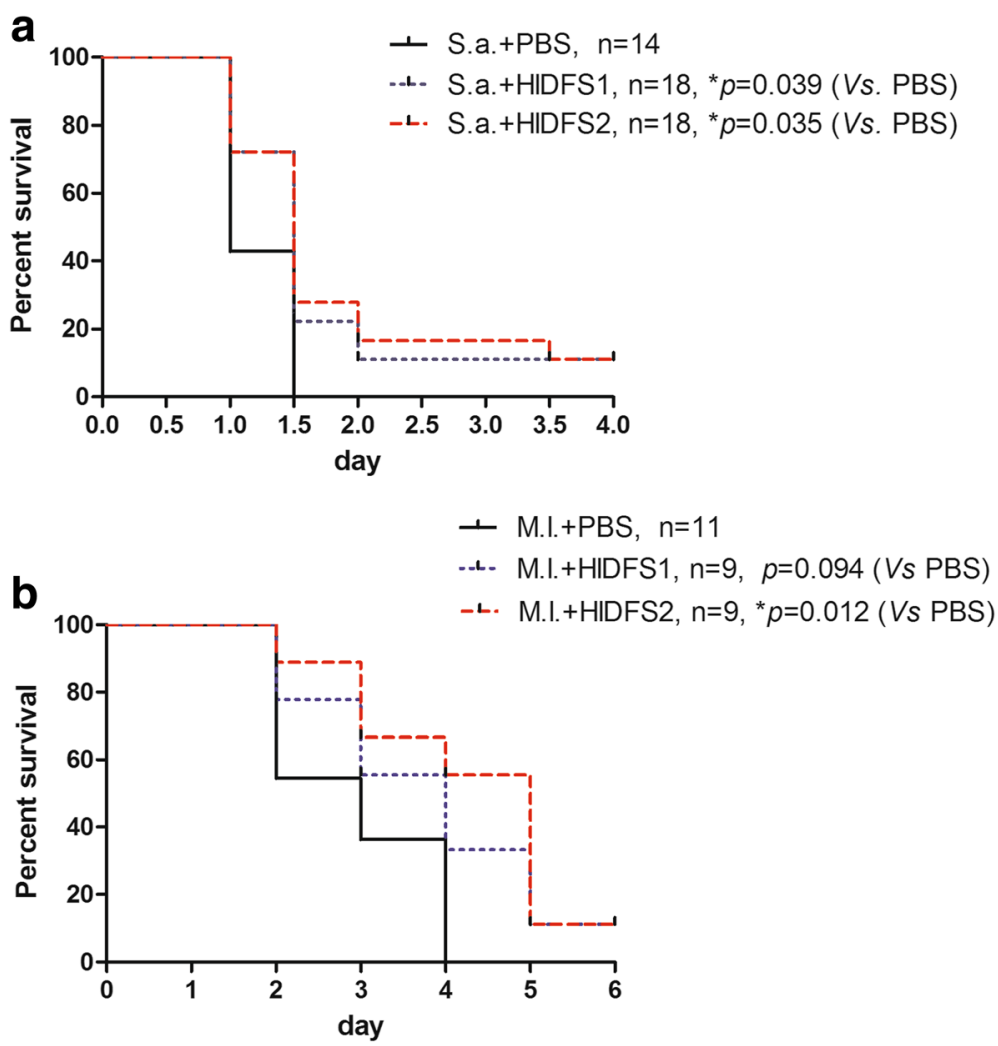

Fig. 4 HIDFS1 and HIDFS2 significantly protect mice against lethal infection of S. aureus and M. luteus. Mice were infected with lethal doses of $M$. luteus (a) $\left(1 \times 10^{6} \mathrm{cfu}\right.$ per C3H/HeJ mouse) and S. aureus (b) $\left(1 \times 10^{8} \mathrm{cfu}\right.$ per C57/BL6 mouse) by i.p. injection. Six hours after infection, mice were treated by i.p. injection of HIDFS1 (100 $\mu \mathrm{g} /$ mouse), HIDFS2 (100 $\mu \mathrm{g} / \mathrm{mouse})$ or saline as controls. Mice were monitored daily for survival and symptoms of disease. Log-rank (Mantel-Cox) tests were used for these survival data

HIDFS1 and HIDFS2 could significantly extended survival time of mice infected by $S$. aureus from 1.5 days to more than 4 days (Fig. 4a). Meanwhile, HIDFS1 and HIDFS2 could extend survival time of mice infected by $M$. luteus from 4 days to more than 6 days (Fig. 4b). These results indicated that HIDFS1 and HIDFS2 had therapeutic efficacy on mice infected by bacteria.

\section{Conclusions}

In conclusion, two functional defensin genes, HIDFS1 and HIDFS2, were identified from H. longicornis of China. Both two defensin isoforms possess bactericidal properties against selective Grampositive and Gram-negative bacteria as well as antibiotic resistant $S$. epidermidis and A. baumannii. Most importantly, HIDFS1 and HIDFS2 had therapeutic efficacy on mice challenged by lethal bacterial infection. These data suggest that HIDFS1 and HIDFS 2 could be safely used in mammalian systems as a potential antimicrobial reagent against various bacteria and other pathogens.

\section{Additional files}

Additional file1: Table S1. Primer sequences for the RT-PCR assay. (DOCX $14 \mathrm{~kb}$ )

Additional file 2: Table S2. The antibiotic resistant information of selective drug resistant bacteria strains. (XLSX $15 \mathrm{~kb}$ )

Additional file 3: Figure S1. HIDFS1 and HIDFS2 inhibited the growth of B. burgdorferi. Quantitative RT-PCR (a) and electrophoresis results (b) for B. burgdorferi flaB gene in DNA samples of spirochete culture. (c) Fluorescence microscopy analysis of GFP signals from B. borgdorferi GFP297 strains. B. borgdorferi GFP-297 is an engineered strain steadily expression GFP protein on the surface of spirochetes. (TIFF $2113 \mathrm{~kb}$ )

Additional file 4: Figure S2. HIDFS1 and HIDFS2 showed no significant antiviral activity against VSV. 293 T cells were infected with VSV-GFP virus at an MOI $=1$ (VSV-GFP virus that expresses GFP as a non-structural protein was provided by Dr. Chunsheng Dong, Soochow University). $20 \mu \mathrm{M}$ HIDFS1 and HIDFS2 or BSA controls were added into the cell culture. $12 \mathrm{~h}$ post-infection, VSV-GFP replication were visualized by GFP signal under the microscope. (TIFF $7201 \mathrm{~kb}$ )

\section{Abbreviations}

AMP: Antimicrobial peptide; CVCC: China Veterinary Culture Collection Center; GFP: green fluorescent protein; HIDFS1: Haemaphysalis longicornis defensin 1; HIDFS2: Haemaphysalis longicornis defensin 2; i.p.: intraperitoneal; ORF: open reading frame; RT-PCR: reverse transcription polymerase chain reaction 


\section{Acknowledgments}

We would like to thank Drs Tingting Feng, Kezhen Wang and Qihan Wu for technical assistance.

\section{Funding}

This work was supported by a Project Funded by the Priority Academic Program Development of Jiangsu Higher Education Institutions, Program for Changjiang Scholars and Innovative Research Team in University (PCSIRT) National Natural Science Foundation of China (81471571, 81271792, and 31400737). The funders had no role in study design, data collection and analysis, decision to publish, or preparation of the manuscript.

\section{Availability of data and materials}

The sequences of HIDFS1 and HIDFS2 CDNA have been deposited in the GenBank database under the accession numbers KY113087 and KY113088. Raw data used and analyzed during the current study are available from the corresponding author on reasonable request.

\section{Authors' contributions}

WP, JW, JZ and JD designed the projects and prepared the manuscript. TS, WP and YS performed all the experiments and analyzed the data. All authors read and approved the final manuscript.

\section{Ethics approval}

All mice were housed in specific pathogen free facilities in accordance with the Guide of National Animal Care and Use committee. All animal experiments were approved by the Institutional Laboratory Animal Care and Use Committee of Soochow University.

\section{Consent for publication}

Not applicable.

\section{Competing interests}

The authors declare that they have no competing interests.

\section{Publisher's Note}

Springer Nature remains neutral with regard to jurisdictional claims in published maps and institutional affiliations.

\section{Author details}

'Institutes of Biology and Medical Sciences, Jiangsu Key Laboratory of Infection and Immunity, Soochow University, Suzhou, People's Republic of China. ${ }^{2}$ Department of Clinical Laboratory, The First Affiliated Hospital of Soochow University, Suzhou, People's Republic of China. ${ }^{3}$ School of Life Science, Fudan University, Shanghai, People's Republic of China.

\section{Received: 12 July 2017 Accepted: 20 September 2017}

\section{Published online: 02 October 2017}

\section{References}

1. Anderson JF, Magnarelli LA. Infect Dis Clin N Am. 2008;22:195-215.

2. Clark RP, Hu LT. Prevention of lyme disease and other tick-borne infections. Infect Dis Clin N Am. 2008;22:381-96.

3. Bulet P, Hetru C, Dimarcq JL, Hoffmann D. Antimicrobial peptides in insects: structure and function. Dev Comp Immunol. 1999;23:329-44.

4. Zasloff M. Antimicrobial peptides of multicellular organisms. Nature. 2002; 415:389-95.

5. Kopacek P, Hajdusek O, Buresova V, Daffre S. Tick innate immunity. Adv Exp Med Biol. 2010;708:137-62.

6. Chrudimska T, Chrudimsky T, Golovchenko M, Rudenko N, Grubhoffer L. New defensins from hard and soft ticks: similarities, differences, and phylogenetic analyses. Vet Parasitol. 2010;167:298-303.

7. Wang J, Bian G, Pan W, Feng T, Dai J. Molecular characterization of a defensin gene from a hard tick, Dermacentor silvarum. Parasit Vectors. 2015; 8:25.

8. Tonk M, Cabezas-Cruz A, Valdes JJ, Rego RO, Rudenko N, Golovchenko M, et al. Identification and partial characterisation of new members of the Ixodes ricinus defensin family. Gene. 2014;540:146-52.

9. Wang $Y$, Zhu S. The defensin gene family expansion in the tick Ixodes scapularis. Dev Comp Immunol. 2011;35:1128-34.
10. Yamaguchi Y, Ouchi Y. Antimicrobial peptide defensin: identification of novel isoforms and the characterization of their physiological roles and thei significance in the pathogenesis of diseases. Proc Jpn Acad Ser B Phys Biol Sci. 2012:88:152-66.

11. Nakajima Y, van der Goes van Naters-Yasui A, Taylor D, Yamakawa M. Antibacterial peptide defensin is involved in midgut immunity of the soft tick, Ornithodoros moubata. Insect Mol Biol. 2002;11:611-8.

12. Johns R, Sonenshine DE, Hynes WL. Response of the tick Dermacentor variabilis (Acari: Ixodidae) to hemocoelic inoculation of Borrelia burgdorferi (Spirochetales). J Med Ent. 2000;37:265-70.

13. Ceraul SM, Sonenshine DE, Ratzlaff RE, Hynes WL. An arthropod defensin expressed by the hemocytes of the American dog tick, Dermacentor variabilis (Acari: Ixodidae). Insect Biochem Mol Biol. 2003:33:1099-103.

14. Liu L, Dai J, Zhao YO, Narasimhan S, Yang Y, Zhang L, Fikrig E. Ixodes scapularis JAK-STAT pathway regulates tick antimicrobial peptides, thereby controlling the agent of human granulocytic anaplasmosis. J Infect Dis. 2012;206:1233-41.

15. Nakajima Y, Ishibashi J, Yukuhiro F, Asaoka A, Taylor D, Yamakawa M. Antibacterial activity and mechanism of action of tick defensin against Gram-positive bacteria. Biochim Biophys Acta. 2003;1624:125-30.

16. Cabezas-Cruz A, Tonk M, Bouchut A, Pierrot C, Pierce RJ, Kotsyfakis M, et al. Antiplasmodial activity is an ancient and conserved feature of tick defensins. Front Microbiol. 2016;7:1682.

17. Meng Z, Jiang LP, Lu OY, Cheng SY, Ye JL, Zhan L. Detection of co-infection with Lyme spirochetes and spotted fever group rickettsiae in a group of Haemaphysalis longicornis. Zhonghua Liu Xing Bing Xue Za Zhi. 2008;29: 1217-1220 (In Chinese).

18. Lee MJ, Chae JS. Molecular detection of Ehrlichia chaffeensis and Anaplasma bovis in the salivary glands from Haemaphysalis longicornis ticks. Vector Borne Zoonotic Dis. 2010;10:411-3.

19. Yu XJ, Liang MF, Zhang SY, Liu Y, Li JD, Sun YL, Zhang L, et al. Fever with thrombocytopenia associated with a novel bunyavirus in China. N Engl J Med. 2011:364:1523-32

20. Luo LM, Zhao L, Wen HL, Zhang ZT, Liu JW, Fang LZ, et al. Haemaphysalis longicornis ticks as reservoir and vector of severe fever with thrombocytopenia syndrome virus in China. Emerg Infect Diseases. 2015;21:1770-6.

21. Saito $Y$, Konnai $S$, Yamada $S$, Imamura S, Nishikado $H$, Ito $T$, et al. Identification and characterization of antimicrobial peptide, defensin, in the taiga tick, Ixodes persulcatus. Insect Mol Biol. 2009:18:531-9.

22. Dai J, Wang P, Adusumilli S, Booth CJ, Narasimhan S, Anguita J, Fikrig E. Antibodies against a tick protein, Salp15, protect mice from the Lyme disease agent. Cell Host Microbe. 2009;6:482-92.

23. Alonzo F 3rd, Benson MA, Chen J, Novick RP, Shopsin B, Torres VJ. Staphylococcus aureus leucocidin ED contributes to systemic infection by targeting neutrophils and promoting bacterial growth in vivo. Mol Microbiol. 2012:83:423-35.

24. Monodane T, Kawabata Y, Takada H. Micrococcus luteus cells and cell walls induce anaphylactoid reactions accompanied by early death and serum cytokines in mice primed with muramyl dipeptide. FEMS Immunol Med Microbiol. 1997:17:49-55.

\section{Submit your next manuscript to BioMed Central and we will help you at every step:}

- We accept pre-submission inquiries

- Our selector tool helps you to find the most relevant journal

- We provide round the clock customer support

- Convenient online submission

- Thorough peer review

- Inclusion in PubMed and all major indexing services

- Maximum visibility for your research

Submit your manuscript at www.biomedcentral.com/submit
BioMed Central 